Article

\title{
Increased Inflammatory Markers Detected in Nasal Lavage Correlate with Paranasal Sinus Abnormalities at MRI in Adolescent Patients with Cystic Fibrosis
}

\author{
Jaehi Chung ${ }^{1,2,+}$, Felix Wünnemann $2,3,4,+$, Johanna Salomon ${ }^{2}$, Sébastien Boutin ${ }^{2,5}$, Dario L. Frey ${ }^{2} \mathbb{D}$, \\ Tobias Albrecht ${ }^{2,6}{ }^{(D}$, Cornelia Joachim ${ }^{1}$, Monika Eichinger ${ }^{2,3,4}$, Marcus A. Mall ${ }^{7,8,9} \mathbb{D}^{\text {, Mark }}$ O. Wielpütz ${ }^{2,3,4}(\mathbb{D}$ \\ and Olaf Sommerburg $1,2, * \mathbb{D}$
}

1 Division of Pediatric Pulmonology \& Allergy and Cystic Fibrosis Center, Department of Pediatrics III, University of Heidelberg, Im Neuenheimer Feld 430, 69120 Heidelberg, Germany; Jaehi.Chung@med.uni-heidelberg.de (J.C.); Cornelia.Joachim@med.uni-heidelberg.de (C.J.)

2 Translational Lung Research Center Heidelberg (TLRC), German Center for Lung Research (DZL), 69120 Heidelberg, Germany; Felix.Wuennemann@med.uni-heidelberg.de (F.W.); johanna.salomon@web.de (J.S.); Sebastien.Boutin@med.uni-heidelberg.de (S.B.); DarioLucas.Frey@med.uni-heidelberg.de (D.L.F.); Tobias.Albrecht@med.uni-heidelberg.de (T.A.); Monika.Eichinger@med.uni-heidelberg.de (M.E.); Mark.Wielpuetz@med.uni-heidelberg.de (M.O.W.)

check for updates

Citation: Chung, J.; Wünnemann, F.; Salomon, J.; Boutin, S.; Frey, D.L.; Albrecht, T.; Joachim, C.; Eichinger, M.; Mall, M.A.; Wielpütz, M.O.; et al. Increased Inflammatory Markers Detected in Nasal Lavage Correlate with Paranasal Sinus Abnormalities at MRI in Adolescent Patients with Cystic Fibrosis. Antioxidants 2021, 10, 1412. https://doi.org/10.3390/ antiox10091412

Academic Editor:

Alessandra Napolitano

Received: 17 August 2021

Accepted: 30 August 2021

Published: 3 September 2021

Publisher's Note: MDPI stays neutral with regard to jurisdictional claims in published maps and institutional affiliations.

Copyright: (๑) 2021 by the authors. Licensee MDPI, Basel, Switzerland. This article is an open access article distributed under the terms and conditions of the Creative Commons Attribution (CC BY) license (https:// creativecommons.org/licenses/by/ $4.0 /)$.
Department of Diagnostic and Interventional Radiology, University Hospital of Heidelberg, 69120 Heidelberg, Germany

4 Department of Diagnostic and Interventional Radiology with Nuclear Medicine, Thoraxklinik at University Hospital of Heidelberg, 69126 Heidelberg, Germany

5 Department of Infectious Diseases, Medical Microbiology and Hygiene, University Hospital of Heidelberg, 69120 Heidelberg, Germany

6 Department of Otorhinolaryngology, Head and Neck Surgery, University Hospital of Heidelberg, 69120 Heidelberg, Germany

7 Department of Pediatric Pulmonology, Immunology and Critical Care Medicine and Cystic Fibrosis Center, Charite-Universitätsmedizin Berlin, 13353 Berlin, Germany; marcus.mall@charite.de

8 Berlin Institute of Health (BIH), 10178 Berlin, Germany

9 German Center for Lung Research (DZL), Associated Partner Site, 13353 Berlin, Germany

* Correspondence: Olaf.Sommerburg@med.uni-heidelberg.de; Tel.: +49-6221-5638790

+ These authors contributed equally as first authors.

\begin{abstract}
Chronic rhinosinusitis (CRS) is a characteristic feature of cystic fibrosis (CF) multiorgan disease and develops early in the life of patients with CF. The study aimed to correlate the inflammatory markers and the presence of structural abnormalities detected by MRI in the paranasal sinuses of patients with CF. Methods: Nasal lavage and MRI of the paranasal sinuses was performed in a cohort of $30 \mathrm{CF}$ patients (median age $14 \mathrm{y}$; range 7-20 y). Morphological abnormalities characteristic of CF were evaluated with a dedicated CRS MRI scoring system and correlated with different inflammation parameters measured in nasal lavage. Inflammation of the paranasal sinuses was positively associated with structural abnormalities in MRI. The concentration of the pro-inflammatory markers neutrophil elastase (NE) and the neutrophil elastase/alpha1-antitrypsin (NE/A1AT) complex correlated significantly with CRS-MRI sum score $(p<0.05, r=0.416$ and $p<0.05, r=0.366$, respectively). $S$. aureus infection was associated with the increased pro-inflammatory cytokine activity of IL-6 and IL-8, and increased levels of NE/A1AT complex in our patients ( $p<0.05$, respectively). CRS-MRI sum score and individual sinus MRI scores were positively associated with inflammatory activity as a sign of CRS pathology present in CF.
\end{abstract}

Keywords: cystic fibrosis; inflammation; Staphylococcus aureus; chronic rhinosinusitis; paranasal sinus; magnetic resonance imaging 


\section{Introduction}

Cystic fibrosis (CF) is one of the most common hereditary diseases in the Caucasian population. Although it is a multiorgan disorder causing symptoms such as pancreatic insufficiency, intestinal malabsorption, infertility and abnormal sweat [1], the leading cause of morbidity and mortality is progressive lung destruction, characterized by mucus obstruction of the small airways, bronchiectasis, neutrophil-dominated inflammation and chronic bacterial infection [2]. The cystic fibrosis transmembrane conductance regulator (CFTR) gene encodes for a cAMP-regulated ion channel that is responsible for the adequate secretion of chloride and bicarbonate in epithelial cells and regulates the epithelial $\mathrm{Na}+$ channel (ENaC) [3]. Mutations in the CFTR gene, resulting in decreased CFTR activity, cause impairments in the hydration of the airway's epithelial surface with highly viscous mucus, which limits mucociliary clearance in the airways, leading to the infection and inflammation of airway tissues $[4,5]$.

Besides chronic lung disease, CF is also characterized by upper airway affection, such as chronic rhinosinusitis (CRS), beginning in the first years of life [4,6]. It is believed that the highly viscous mucus in CF obstructs the ostia of the sinuses, leading to chronic bacterial infection and inflammation [7]. Chronic inflammation in the sinuses is believed to promote goblet cell hyperplasia and squamous cell metaplasia, causing remodeling processes [8,9]. The clinical symptoms of CRS in CF include nasal congestion, rhinorrhea, nasal polyps, anosmia and sleep disorder [10]. In patients with $\mathrm{CF}$, paranasal sinuses can serve as niches for bacteria, such as Staphylococcus aureus (S. aureus), Haemophilus influenzae (H. influenzae) and Pseudomonas aeruginosa (P. aeruginosa), to inhabit the sinuses and evolve into highly pathogenic microorganisms [11-15], which, in unstable phases, may migrate and play a role in recurrent infections and the inflammatory processes of the lower airways [12,16].

However, the exact relationship of chronic inflammation in the upper airways with the development of structural abnormalities of the paranasal sinuses is not well understood. After bacterial colonization, IL- 8 and TNF- $\alpha$ as chemoattractants induce the migration of neutrophils into the airways [17]. When stimulated, neutrophils release free radicals produced via NADPH or myeloperoxidase as a mechanism for killing pathogens $[18,19]$. In $\mathrm{CF}$, neutrophil-dominated inflammation results in the overproduction of reactive oxygen species (ROS), leading to oxidative stress with increased lipid and protein peroxidation [20,21]. Additionally, large amounts of neutrophil elastase (NE) are released from azurophilic granules of the neutrophils [22]. NE has important protective functions, such as remodeling the extracellular matrix and cleaving pathogens [23]. Antiproteases, such as alpha1-antitrypsin (A1AT) and secretory leukocyte protease inhibitor (SLPI), are the physiological antagonists of NE and protect the airway tissues [23-25]. However, the permanent imbalance between oxidants and antioxidants in the inflammatory process in the paranasal sinuses of patients with CF leads to a situation in which irreversible structural damage to the surrounding tissues of the upper airways occurs. For the estimation of the inflammatory status of the paranasal sinuses in patients with CF, nasal lavage has been shown to be a good noninvasive and safe method for obtaining sufficient sample material [26-28].

The onset of the development of structural abnormalities in the paranasal sinuses of patients with CF appears very early, as the increased opacity of the paranasal sinuses in those patients can be seen on radiographs as early as 8 months of age [29]. In recent years, however, it has been shown that magnetic resonance imaging (MRI) of the head, with its excellent soft tissue differentiation capability, can quickly and reliably identify characteristic abnormalities of CRS, such as mucosal swelling, mucopyoceles, and nasal polyps in the sinuses of patients with $\mathrm{CF}[6,30]$. Therefore, such an examination should be performed in patients with $\mathrm{CF}$ at regular intervals for diagnosis and therapy management.

To the best of our knowledge, the inflammation of the upper airways has not been studied in association with morphological changes via MRI of the paranasal sinuses in patients with CF before. Therefore, this pilot study aimed to correlate the presence of struc- 
tural abnormalities and the CRS-MRI score of the paranasal sinuses with the inflammatory status measured in the nasal lavage supernatant in patients with CF.

\section{Materials and Methods}

\subsection{Study Population}

This project was conducted as a cross-sectional sub-study within a prospective longitudinal observational study in patients with CF at the CF center in Heidelberg, Germany. Informed written consent was obtained from the patients or their parents or legal guardians. Both the sub-study reported in the manuscript and the above-mentioned overarching prospective longitudinal observational study were approved by the ethics committee of the Medical Faculty of the University of Heidelberg (S-175/2020 and S-370/2011, respectively). Between February 2019 and February 2020, all CF patients of the CF Center Heidelberg who had received their annual check-up with clinical assessment, lung function tests, throat and nasal swabs, and, when possible, lavages and MRIs of the paranasal sinuses were included in the study. Age over 7 years was considered to be necessary for performing the nasal lavage correctly, according to the protocol. All CF patients received therapy according to the current guidelines. The exclusion criteria for this study were acute exacerbation at the time of the study visit (yearly check-up and/or MRI) or surgery performed on the paranasal sinuses or the nose within 24 months before the study. The MRI was performed on all patients on the same day of the yearly check-up, or within an interval up to 4 months before or after sampling the upper airway with nasal lavage and nose swabs.

\subsection{Sample Collection and Measurement of Inflammatory Parameters}

Nasal swabs were collected in patients with CF from the anterior nares first for microbiological culture, and these were processed by the local microbiology laboratory according to German quality assurance guidelines for CF microbiology [31]. Afterwards, nasal lavage was performed in these patients with clinically stable disease. The nasal lavage fluid was obtained by rinsing 5 to $10 \mathrm{~mL}$ isotonic saline $(0.9 \% \mathrm{NaCl}$, Braun, Melsungen, Germany) into each nostril, according to Beiersdorf et al. (2013) [26]. The patients were asked to hold the fluid for a few seconds in a reclined head position, then lean forward and exhale it. The fluid was collected in a sterile beaker. Lavage samples were centrifuged (300 rpm, $\left.10 \mathrm{~min}, 4^{\circ} \mathrm{C}\right)$. The supernatant was aliquoted and stored at $-80^{\circ} \mathrm{C}$. Viable cells were pelleted (300 rpm, $10 \mathrm{~min}, 4^{\circ} \mathrm{C}$ ), resuspended and counted with a hemocytometer. Total protein concentration was measured using $25 \mu \mathrm{L}$ of supernatant (Pierce BCA Protein Assay Kit, Thermo Fisher Scientific, Rockford, IL, USA) at a $562 \mathrm{~nm}$ wavelength. The concentration of the inflammation markers IL-1 $\beta$, IL-6, IL- 8 and TNF- $\alpha$ in the NL supernatant was determined with a bead-based immunoassay using flow cytometry (Cytometric Bead Arrays, BD Biosciences, San Diego, CA, USA). The NE/A1AT complex, SLPI and TIMP-1 were measured in duplicates using ELISAs (Human PMN-Elastase ELISA Kit, Invitrogen, Quantikine ELISA Human SLPI Immunoassay R\&D Systems, Inc., Minneapolis, MN, USA, Quantikine Elisa Human TIMP-1 Immunoassay, R\&D Systems, Inc., Minneapolis, MN, USA). The activity of free soluble neutrophil elastase was detected from the cell-free supernatant of nasal lavage probes with the FRET-based reporter NEmo-1 (Sirius Fine Chemicals, Bremen, Germany) [32,33]. All inflammatory mediators were normalized to the total protein concentration.

\subsection{MRI and Image Assessment}

MRI of the sinuses from CF patients was performed on a clinical 1.5-T scanner (Magnetom Avanto, Siemens Healthineers, Erlangen, Germany) as previously described [6]. Native T1- and T2-weighted images were obtained. After the application of intravenous gadolinium-based contrast, T1-weighted images were acquired (Dotarem, Guerbet AG, Sulzbach, Germany). MRI scans were assessed with a CRS MRI scoring system evaluating all paranasal sinuses regarding sinus dimensions, degree of opacification, and specific abnormalities such as mucosal swelling, mucopyoceles, polyps and effusion [6]. Further, 
the deformation of the semilunar hiatus in the maxillary sinus was graded. The maximal CRS-MRI score is 68 [6].

\subsection{Lung Function Test}

Lung function variables were used to describe the clinical conditions of the patients in our cohort. The Forced Expiratory Volume in one second as a percent of the predicted value (FEV1\%pred.) was determined with spirometry, and calculated according to reference values from the European Respiratory Society [34]. In addition, the lung clearance index (LCI), as a parameter to measure ventilation inhomogeneities in the small airways, was determined additionally with a multiple breath washout (MBW) test with $\mathrm{N}_{2}$ as a tracer gas [35,36]. The normal values for the LCI $2.5 \%$ (hereafter referred to only as LCI) we used are reported to range from 6.16 to 7.91 for school-aged children 6 to 18 years of age [37]. The higher the LCI value, the worse the airway ventilation due to mucus-related obstruction.

\subsection{Data Analysis}

As part of the evaluation, the inflammation parameters measured in the nasal lavage should be evaluated together with the results of the MRI of the paranasal sinuses. For this purpose, the presence of certain sinonasal pathologies as well as the CRS MRI score should be correlated to individual inflammation parameters. Furthermore, the microbiological colonization of the upper airways as well as the results of the two lung function tests were included in the evaluation. The data were analyzed using GraphPad Prism 6 (Graph Pad software Inc., San Diego, CA, USA). The results are presented as mean \pm standard deviation when distributed normally. Non-normally distributed variables were presented as median values with minimum and maximum values. The influence of the bacterial colonization on the inflammatory response was measured with the Mann-Whitney-U-test. The Spearman correlation coefficient was calculated for the correlation of inflammatory parameters and CRS-MRI scores, as well as single pathologies. A p-value below 0.05 was considered statistically significant.

\section{Results}

This study was conducted as a sub-study of an ongoing longitudinal study, in which nasal lavage samples were taken to monitor inflammatory parameters. The recruitment of study participants from patients at our CF center is shown in Figure 1. At the time of the MRI sub-study, 36 of the 135 patients with $\mathrm{CF}$ from our $\mathrm{CF}$ center were participating in the longitudinal study performing nasal lavages. After reviewing the inclusion and exclusion criteria, 5 patients had to be excluded for the MRI sub-study, so 31 patients with cystic fibrosis (median age 14 years, range 7-20 years) in a stable clinical condition were included. Clinical symptoms of sinonasal disease were present in 17 of these 31 . Of those 17 patients, 6 had undergone sinus surgery or resection of polyps, but this was more than 24 months before the start of the study. Due to motion artefacts, the MRI of one patient had to be excluded from the statistical analysis. Thus, in the end, 30 patients were included in this study. Although this is only a sub-cohort of all patients with $\mathrm{CF}$, it mirrors a representative cross-section of patients from our CF center, with 12 patients homozygous for F508del, 14 patients heterozygous for F508del, and 4 patients with other CFTR mutations. For further information on clinical characteristics, refer to Table 1.

The upper airways of the CF patients in our cohort were vastly colonized with Coagulase-negative Staphylococcus (CoNS) (79.3\%). In more than one third of the patients, the $\mathrm{CF}$ typical pathogen S. aureus (37.9\%) was found in the upper airways. Only a few were colonized with Corynebacterium species, Moraxella catarrhalis or Streptococcus species. None of the patients with CF in our cohort were colonized with P. aeruginosa or H. influenzae in the nasal swabs. Further information on the bacterial colonization in our cohort can be found in the online supplement in Table S1. 


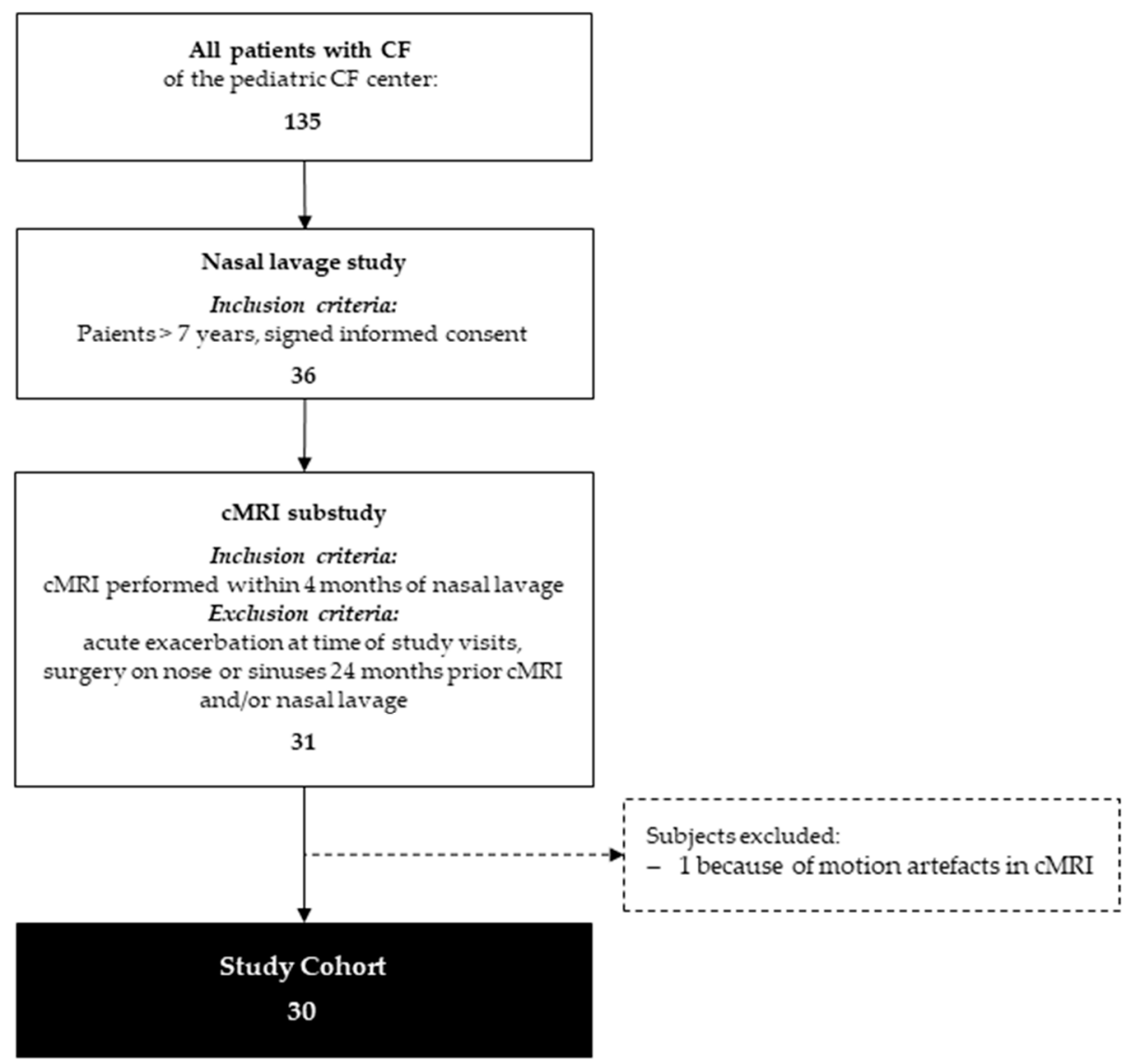

Figure 1. Patient recruitment.

Table 1. Clinical characteristics of the study population, $n=30$.

\begin{tabular}{ccc}
\hline Clinical Parameter & Median (Range) & Mean \pm SD \\
\hline Age (years) & $14(7-20)$ & $13.7 \pm 3.3$ \\
Sex, female, $n(\%)$ & $10(33.3 \%)$ & $19.3 \pm 3.0$ \\
BMI $\left(\mathrm{kg} / \mathrm{m}^{2}\right)(n=29)$ & $19.4(14.6-24.1)$ & \\
CFTR genotype & $12(40.0 \%)$ & \\
F508del/F508del, $n(\%)$ & $14(46.7 \%)$ & \\
F508del/other, $n(\%)$ & $4(13.3 \%)$ & \\
Other/other, $n(\%)$ & $14(46.7 \%)$ & \\
CFTR modulator therapy, $n(\%)$ & $83.7(25.2-121.2)$ & \\
FEV1\%pred. $(n=30)$ & $8.5(5.5-18.0)$ & \\
LCI $(n=28)$ & & \\
Nasal swabs $(n=29)$ & $23(79.3 \%)$ & \\
Coagulase negative Staphylococcus, $n(\%)$ & $11(37.9 \%)$ & \\
Staphylococcus aureus, $n(\%)$ & $2(6.9 \%)$ & \\
Moraxella catarralis, $n(\%)$ & $7(24.1 \%)$ & \\
Corynebacterium sp., $n(\%)$ & $0(0 \%)$ & \\
Pseudomonas aeruginosa, $n(\%)$ & $0(0 \%)$ & \\
Haemophilus influenzae, $n(\%)$ & & \\
\hline Abber $(\%)$ & & \\
\hline
\end{tabular}

Abbreviations: SD = standard deviation, BMI = body mass index, FEV1\%pred. = forced expiratory volume in one second in percent predicted, $\mathrm{LCI}=$ lung clearance index, sp. = species. If a dataset was incomplete for a certain parameter, the number of patients for the respective parameter is given in parenthesis.

\subsection{Inflammation Measured in Nasal Lavage}

We were able to obtain sufficient material in the nasal lavage samples from all patients in this study. The patients showed a varying concentration of vial cells, ranging from 1750 to 772,500 cells $/ \mathrm{mL}$. Accordingly, different amounts of total protein were shown, but the quantities were always sufficient to perform the determination of the inflammatory 
parameters. A complete overview of the results on the inflammatory parameters of the 30 patients with CF in the study is shown in Table 2.

Table 2. Inflammatory parameters, $n=30$.

\begin{tabular}{|c|c|}
\hline Inflammatory Parameter & Median (Range) \\
\hline Total Cells/mL & $14,500(1750-772,500)$ \\
\hline Neutrophils (\%) & $82.61(0-100)$ \\
\hline Total protein level $(\mu \mathrm{g} / \mathrm{mL})$ & $155.5(11.76-415.11)$ \\
\hline IL-1 $\beta(\mathrm{pg} / \mathrm{mL})$ & $1.78(0.10-96.64)$ \\
\hline IL-6 $(\mathrm{fg} / \mathrm{mL})$ & $424.4(0.0-68,392.53)$ \\
\hline IL-8 (pg/mL) & $91.04(21.19-1270.54)$ \\
\hline NE/A1AT complex (ng/mL) & $2.89(0-50.58)$ \\
\hline NE activity $(\mathrm{ng} / \mathrm{mL})$ & $0(0-0.04)$ \\
\hline SLPI (ng/mL) & $192,337.48(14.60-2,217,601.86)$ \\
\hline TIMP-1 (ng/mL) & $3.93(0.02-51.35)$ \\
\hline
\end{tabular}

Abbreviations: IL = interleukin, TNF = tumor necrosis factor, NE = neutrophil elastase, $\mathrm{A} 1 \mathrm{AT}=$ alpha-1-antitrypsin, SLPI = secretory leukocyte protease inhibitor, TIMP-1 = tissue inhibitor of metalloprotease- 1 .

\subsection{Influence of Microbiological Colonization in the Iinflammatory Response}

The results of nasal microbiological swabs corresponding to the time of nasal lavage were available for 29 of the 30 patients included in the study. Colonization with S. aureus was associated with higher inflammation. In patients with S. aureus infection of the upper airways, pro-inflammatory cytokines, such as IL-8 $(p=0.037)$ and IL-6 $(p=0.021)$, and also the NE/A1AT complex $(p=0.042)$, were significantly higher than in patients without. However, other proinflammatory cytokines and antiproteases, such as SLPI or TIMP, were also increased, but this difference was not statistically significant. The results for the cytokine levels of IL-6 and IL-8 as well as for the NE/A1AT complex and TIMP-1 are shown in Figure 2.
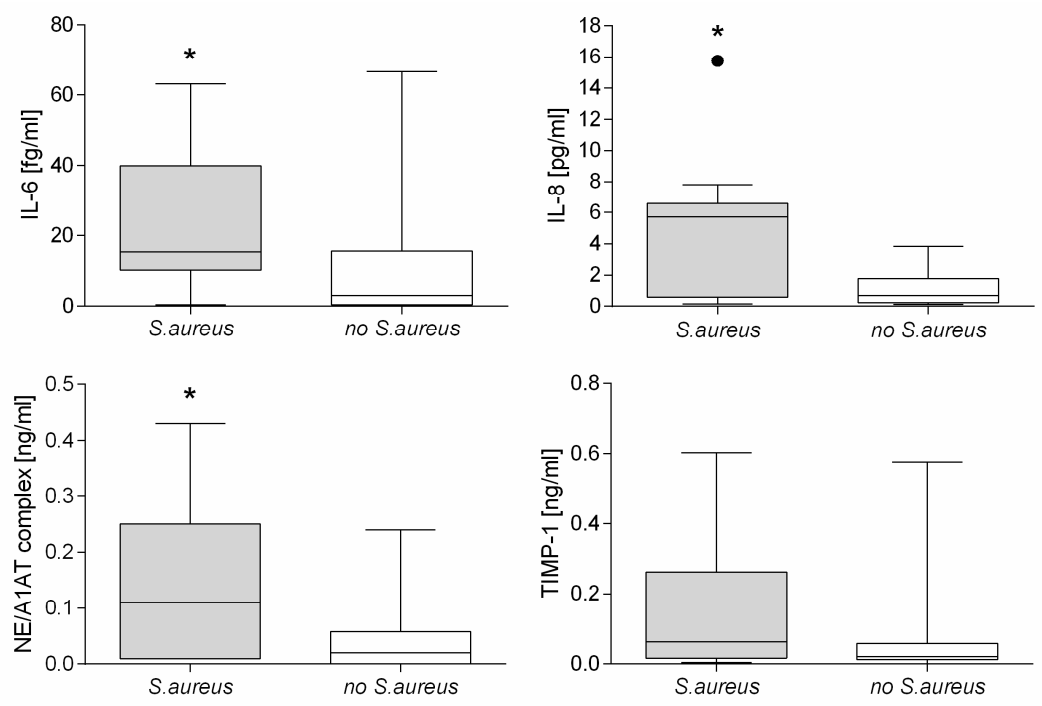

Figure 2. Comparison of the groups of patients with $\mathrm{CF}$ colonized with and without $S$. aureus for the inflammatory parameters IL-6, IL-8, the NE/A1AT complex and TIMP-1. Cytokine levels of IL-6 and IL-8, as well as of the NE/A1AT complex, were found to be significantly increased in patients with CF with colonization of $S$. aureus $\left({ }^{*} p<0.05, \bullet\right.$ outlier $)$.

For clarity, Table S1 in the online supplement lists the individual results of the 29 patients considered here for nasal bacterial colonization, CRS-MRI score, and inflammatory parameters. 


\subsection{Sinus Abnormalities Detected by MRI}

Mucosal swelling was highly prevalent in the maxillary (95\%), sphenoid (87\%) and ethmoid sinuses $(100 \%)$. Mucopyoceles were observed in the maxillary $(82 \%)$, sphenoid $(60 \%)$ and ethmoid sinuses (58\%). Wall deformities of the maxillary sinus could be detected in $77 \%$ of patients in our cohort. In nine patients of our cohort, either one or both frontal sinuses were not developed. Figure 3 provides an overview of the typical pathologies found in the patients with CF in our study cohort. Table 3 provides an overview of the sinus pathologies seen via MRI in our study participants.

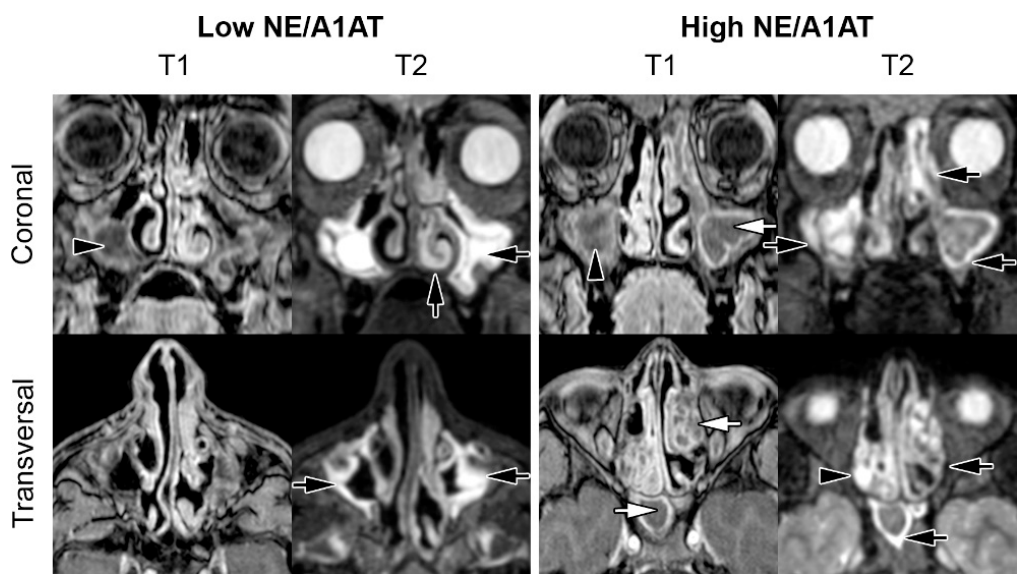

Figure 3. Representative MRI of the paranasal sinuses in patients with $\mathrm{CF}$ with low and high NE/A1AT ratios, respectively. A 16-year-old CF patient with a low NE/A1AT ratio demonstrated mucosal swelling (black arrows) of the maxillary sinus and left inferior concha, accompanied by polyps (black arrowheads) in the right maxillary sinus. The CRS-MRI score was 9. The 14-year-old CF patient with high NE/A1AT ratio also showed ubiquitous and more extensive mucosal swelling, polyps, as well as mucopyoceles (white arrows) of the maxillary, ethmoid and sphenoid sinus. The CRS-MRI score was 40. Abbreviations: $\mathrm{NE}=$ neutrophil elastase, $\mathrm{A} 1 \mathrm{AT}=$ alpha1-antitrypsin.

Table 3. Sinus pathologies seen in MRI, $n=30$.

\begin{tabular}{ccc}
\hline & $\begin{array}{c}\text { Patients with Sinonasal } \\
\text { Pathologies, } \boldsymbol{n} \mathbf{( \% )}\end{array}$ & $\begin{array}{c}\text { CRS-MRI Score } \\
\text { Median (Range) }\end{array}$ \\
\hline CRS-MRI sum score & & $30.5(9-44)$ \\
Maxillary sinus & $28(93.3 \%)$ & $13(3-18)$ \\
Wall deformation & $26(86.7 \%)$ & $2(0-4)$ \\
Mucopyoceles & $29(96.7 \%)$ & $1(0-4)$ \\
Mucosal swelling & $2(6.7 \%)$ & $3(0-4)$ \\
Effusion & $17(56.7 \%)$ & $0(0-2)$ \\
Polyps & & $1(0-3)$ \\
Sphenoidal sinus & $21(70 \%)$ & $8.5(0-12)$ \\
Mucopyoceles & $30(100 \%)$ & $2(0-4)$ \\
Mucosal swelling & $0(0 \%)$ & $3(0-3)$ \\
Effusion & $0(0 \%)$ & $0(0)$ \\
Polyps & & $0(0)$ \\
Ethmoidal sinus & $18(60 \%)$ & $8.5(6-12)$ \\
Mucopyoceles & $30(100 \%)$ & $2(0-4)$ \\
Mucosal swelling & $0(0 \%)$ & $4(2-4)$ \\
Effusion & $0(0 \%)$ & $0(0)$ \\
Polyps & & $0(0)$ \\
Mastoid cells & $0(0 \%)$ & $0(0-5)$ \\
Mucopyoceles & $3(10 \%)$ & $0(0)$ \\
Mucosal swelling & $1(3.3 \%)$ & $0(0-2)$ \\
Effusion & $0(0 \%)$ & $0(0-2)$ \\
Polyps & & $0(0)$ \\
\hline
\end{tabular}




\subsection{Inflammatory Parameters and MRI Scores}

The CRS MRI sum score correlated to all measured inflammatory parameters in the nasal lavage. Moreover, it correlated significantly to the NE/A1AT complex $(p<0.05$, $r=0.366)$ and to the neutrophil elastase activity $(p<0.05, r=0.416)$. Additionally, it could be seen that higher CRS-MRI subscores were positively associated with an increase in inflammatory markers. The score of the ethmoidal sinus correlated significantly to the number of neutrophils $(p<0.05, r=0.411)$ and to the neutrophil elastase activity $(p<0.01$, $r=0.468$ ). Greater inflammation led to more radiographic changes associated with CRS being detected in the sinuses. SLPI, as a protective antiprotease, correlated inversely with the presence of wall deformations in the maxillary sinus $(r=-0.313)$, but not significantly. A complete overview of the correlations between the measured inflammation parameters and the MRI scores is shown in Figure 4.

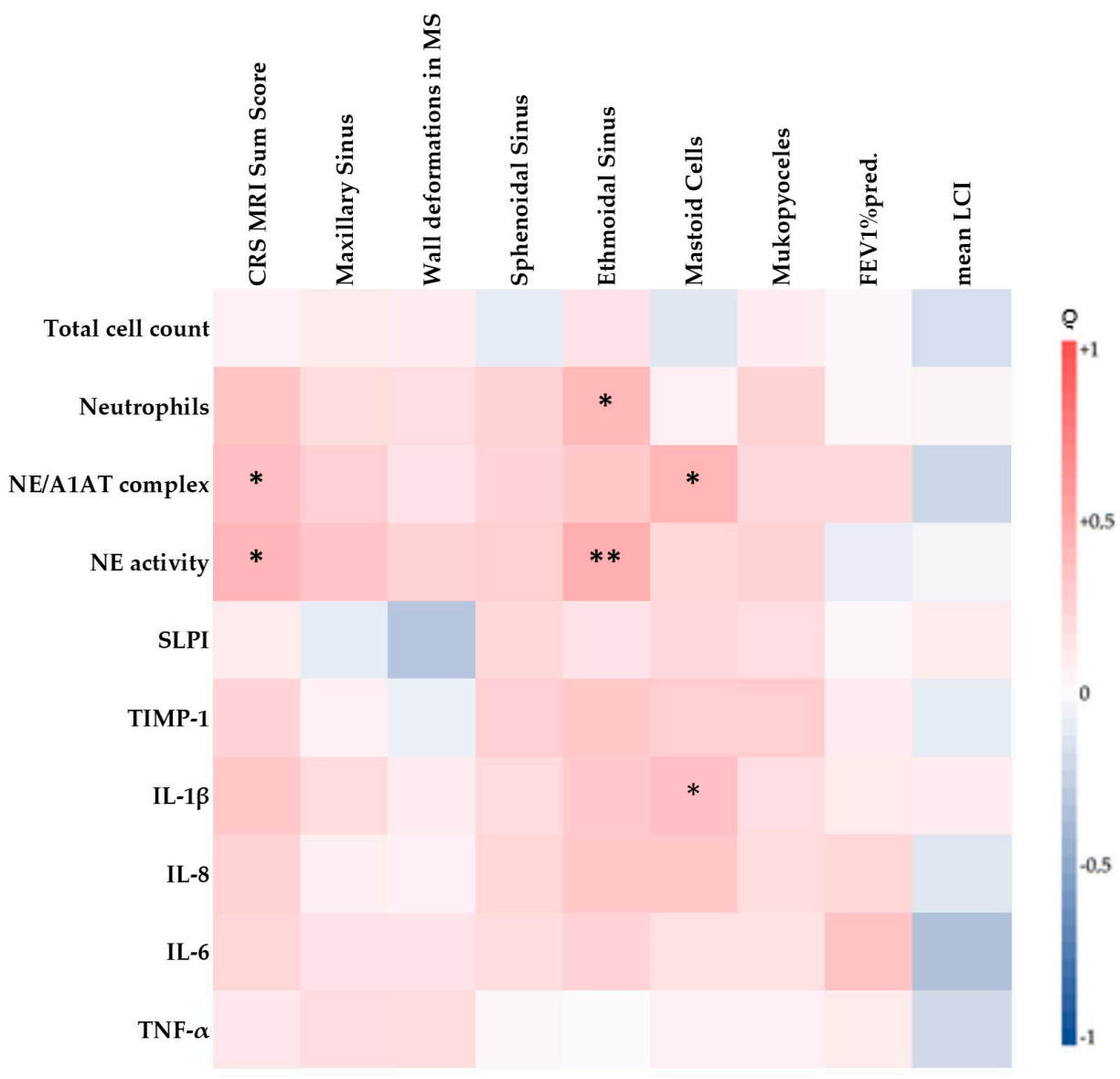

Figure 4. Spearman correlation of inflammatory parameters (NE/A1AT complex, SLPI, TIMP-1, IL1 $\beta$, IL-6, IL-8, TNF- $\alpha$ ) to MRI-scores of the sinuses and clinical parameters (pathogen colonization and lung function (FEV1\%pred. and mean LCI)). ${ }^{*} p<0.05 ;{ }^{* *} p<0.01$. Abbreviations: MS $=$ maxillary sinus.

\subsection{Influence of Sinonasal CF Disease on Lung Function}

The values for the FEV1\%pred. in our patient cohort ranged from 25 to $121 \%$. The median was $84 \%$, indicating that the majority of patients had normal FEV1 values. LCI values ranged from 5.53 to 18.04 , with a median of 8.46 , slightly above the cutoff level for this age group. No significant relation has been seen in our patient cohort between lung function estimated by FEV1\%pred. and mean LCI and the inflammatory markers measured in nasal lavage (Figure 4), or morphological changes seen in MR imaging. 


\section{Discussion}

Sinus involvement is part of the multiorgan disease in patients with CF. Radiologic abnormalities associated with sinus disease are known to start early in the lives of CF patients. In our study, we demonstrated that increased inflammatory parameters in nasal lavage specimens from patients with $\mathrm{CF}$ correlate with morphologic changes and structural abnormalities in the paranasal sinuses seen on MRIs performed at that time.

\subsection{Inflammation and Infection of the Upper Airways}

Our results show a significant increase in the inflammatory activity in the upper airways of patients with CF. The measured levels for the individual inflammatory parameters are, in principle, consistent with the results of previous studies measuring inflammatory markers in nasal lavages $[26,27,38-40]$. Nevertheless, our values for cytokines seem to be somewhat lower compared to others. One reason for this might be the younger age in our cohort compared, e.g., to that published by Hentschel et al. [27]. However, our results reflecting cytokine activity still differ significantly from values measured for healthy subjects [27]. In this context, it is interesting to note that the concentration of antiproteases measured in our cohort was higher than that measured by other authors. This may confirm our assumption that the younger patients in our study cohort were clinically more stable than the older patients previously studied in other cohorts $[26,27,39]$. Another explanation for the lower inflammatory response could be that colonization with pathogenic bacteria was not yet as pronounced in the patients in our cohort.

In our cohort, 11 patients (38\%) were colonized with the typical CF pathogen S. aureus, which was associated with higher levels of inflammatory cytokines, neutrophil elastase and protective antiproteases. The neutrophil-dominated immune response, with the release of pro-inflammatory cytokines, usually leads to high oxidative stress in the airways. High concentrations of NE, released by neutrophils, overwhelm the antiprotease capacity and contribute to the destruction of the airway's surface cells by cleaving antiproteases and also digesting the extracellular matrix [41,42]. Further, the ROS produced by leukocytes during phagocytosis can inactivate $\mathrm{A} 1 \mathrm{AT}$, and as a result interfere, in the protease/antiprotease ratio [43,44]. However, in patients in whom Corynebacterium sp. $(n=7,24 \%)$ was detected in the upper respiratory tract, this colonization did not lead to such an inflammatory reaction as we had seen for S. aureus. Most of our patients (79\%) were colonized with CoNS. Colonization with CoNS showed no significant influence on all of the inflammatory parameters, highlighting its role as a commensal bacterium, as also seen in other studies [45]. None of our patients were colonized with P. aeruginosa or $H$. influenzae. This also distinguishes our cohort from older patients with $\mathrm{CF}$, in whom upper airway colonization was previously studied in relation to inflammatory activity $[13,15]$.

In general, inflammatory responses in the upper airways, as seen in our data for S. aureus, can also lead to irreversible tissue damage, and reduced lung function in the lower airways if the airways are thought of as a single organ. However, in our cohort, no association was found between upper airway inflammation and lung function as a global parameter for the condition of a CF patient. This was true not only for FEV1\%pred., but also for LCI, which reflects ventilatory inhomogeneities due to mucus in the airways. Future studies will show whether there really is no correlation, or whether it could not be demonstrated because of our study design or the relatively healthy cohort.

\subsection{Inflammation and Structural Abnormalities in the MRI}

In a recent paper published by our group, it could be shown that the prevalence and severity of MRI abnormalities associated with CRS in CF increase with age. Accordingly, the CRS-MRI scores obtained in our present cohort were higher than in the cohort previously described in patients with CF ranging from 0 to 6 years of life [6]. Other previously published studies on patients with CF using CT reported an increased prevalence of aplasia, especially of the frontal sinuses, and hypoplasia of the maxillary and the sphenoid sinus in adolescent and adult patients with CF [46-48]. In the present study, we can confirm 
a higher rate of aplasia and hypoplasia of the frontal sinus in our patient cohort. However, the delays in sinus development in patients with $\mathrm{CF}$ remain poorly understood. According to our previously published data, mucosal swelling is the earliest sign of CF-related sinus disease, and is detectable as early as the first few months of life [6]. Mucosal swelling likely reflects inflammation, similarly to wall thickening of the lower airway, and may be reversible $[49,50]$. In addition, mucopyoceles seem to be a characteristic feature of $\mathrm{CF}$ and, when present, also dominate the morphologic changes in the paranasal sinuses. Our current results also demonstrate that inflammation of the paranasal sinuses may influence the structural changes characteristic of CRS, as the CRS-MRI subscores of all sinuses and the CRS-MRI sum score correlated positively with the measured inflammation parameters. This suggests that the higher the inflammation, the greater the morphologic changes in the paranasal sinuses. Previous studies found P. aeruginosa in the aspirates of mucopyoceles from the maxillary sinus $[46,48]$. Similar to lower respiratory tract mucus plugging, mucus retention in the paranasal sinuses may serve as a nidus for chronic infections and a reservoir for CF pathogens. In our present cohort of mostly school-aged CF patients, no nasal colonization with $P$. aeruginosa could be detected; however, those patients colonized with $S$. aureus showed significantly higher inflammatory parameters as well, and a trend, albeit not significant, toward an increased CRS-MRI sum score. The CRS-MRI sum score as well as the CRS-MRI subscore of the ethmoid sinus were positively associated with NE activity. This might indicate that the higher protease activity is related to destruction of the surrounding tissues of the corresponding paranasal sinuses. In this respect, it is likely that the colonization of the paranasal sinuses with typical CF pathogens triggers inflammation and oxidative stress, and that the structural abnormalities of the paranasal sinuses influence each other. Nasal polyps, also typical of cystic fibrosis, represent irreversible structural abnormalities caused by the benign hyperproliferation of chronically inflamed mucosal tissue, and may also be discussed in this context.

\subsection{Limitations}

Our study has several limitations. First, our cohort consists of patients with CF aged 7 to 20 years. With a median age of 14 years, it consists mainly of adolescents, and may relatively healthy compared with cohorts of previous studies. Second, because participation in the overarching longitudinal study performing nasal lavage was voluntary, not all patients with $\mathrm{CF}$ from our $\mathrm{CF}$ center who were eligible for this study participated. On the one hand, this could lead to a potential distortion of the study cohort, as it may not be as representative as originally thought. On the other hand, this may lead to a bias, as more therapy-adherent parents and patients may have consented to our sub-study, and thus influenced our cohort accordingly. Third, the cell and protein contents of our nasal lavage samples show significant interindividual differences. Although we can say, based on experience in determining inflammation parameters in our laboratory, that we remained above the minimum amount of material to be studied in each case, we obtained very low concentrations or activities in individual inflammatory parameters in some patients. Fourth, our study would have benefited if the subjective well-being of the participating patients had been additionally evaluated with a sinonasal outcome score (SNOT) [51]. However, to the best of our knowledge, the current most used SNOT-22 test [52] has not yet been validated for patients with CF. To assess treatment effects on CF-related CRS, especially of the new CFTR modulators, future studies evaluating an appropriate SNOT score are essential.

\section{Conclusions}

Inflammation of the paranasal sinuses is positively associated with structural abnormalities on MRI. The CRS-MRI sum score and the subscores for individual sinuses were positively associated with NE activity, suggesting that higher protease activity is related to CRS. We also found that upper respiratory tract infection with S. aureus was associated with a more pronounced inflammatory response. Therefore, our data may suggest that 
colonization with the CF-typical pathogen S. aureus upregulates inflammatory activity in the upper airways, thereby possibly influencing the morphological changes characteristic of CRS. Since this was a pilot study performed on a voluntary basis, further studies with a larger cohort and wider age range should be performed to evaluate the relation of infection, inflammation, and the pathogenesis of CRS in more detail.

Supplementary Materials: The following is available at https://www.mdpi.com/article/10.3390/ antiox10091412/s1, Table S1: An overview of the bacterial colonization of the study population with the corresponding CRS MRI sum score, $n=29$.

Author Contributions: Conception and design of the study: O.S., M.O.W., J.C., F.W. and M.E.; acquisition, analysis and interpretation of data: J.C., F.W., C.J., J.S., S.B., D.L.F., O.S., M.O.W. and M.E.; drafting the article or revising it critically for important intellectual content: J.C., F.W., C.J., J.S., S.B., D.L.F., T.A., M.E., O.S., M.O.W. and M.A.M. All authors have read and agreed to the published version of the manuscript.

Funding: This study was supported in part by grants from the German Federal Ministry of Education and Research (82DZL004A1 and 82DZL009B1).

Institutional Review Board Statement: The study was conducted according to the guidelines of the Declaration of Helsinki and approved by the Institutional Review Board (or Ethics Committee) of the University of Heidelberg (S-370/2011 and S-175/2020).

Informed Consent Statement: This project was performed as part of a prospective longitudinal observational study in patients with CF followed at the CF Center in Heidelberg, Germany, and was approved by the ethics committee of the University of Heidelberg (S-175/2020). Written informed consent was obtained from all patients, their parents or guardians before initiation.

Data Availability Statement: Data are contained within the article and Supplementary Materials.

Conflicts of Interest: All authors declare no conflict of interest.

\section{References}

1. Elborn, J.S. Cystic fibrosis. Lancet 2016, 388, 2519-2531. [CrossRef]

2. Mall, M.A.; Hartl, D. CFTR: Cystic fibrosis and beyond. Eur. Respir. J. 2014, 44, 1042-1054. [CrossRef]

3. Gentzsch, M.; Mall, M.A. Ion channel modulators in cystic fibrosis. Chest 2018, 154, 383-393. [CrossRef] [PubMed]

4. Ramsey, B.; Richardson, M.A. Impact of sinusitis in cystic fibrosis. J. Allergy Clin. Immunol. 1992, 90, 547-552. [CrossRef]

5. Boucher, R.C. Airway surface dehydration in cystic fibrosis: Pathogenesis and therapy. Annu. Rev. Med. 2007, 58, 157-170. [CrossRef]

6. Sommerburg, O.; Wielpütz, M.O.; Trame, J.-P.; Wuennemann, F.; Optazaite, E.; Stahl, M.; Puderbach, M.U.; Kopp-Schneider, A.; Fritzsching, E.; Kauczor, H.-U.; et al. Magnetic resonance imaging detects chronic rhinosinusitis in infants and preschool children with cystic fibrosis. Ann. Am. Thorac. Soc. 2020, 17, 714-723. [CrossRef] [PubMed]

7. Chaaban, M.R.; Kejner, A.; Rowe, S.M.; Woodworth, B.A. Cystic fibrosis chronic rhinosinusitis: A comprehensive review. Am. J. Rhinol. Allergy 2013, 27, 387-395. [CrossRef]

8. Gysin, C.; Alothman, G.A.; Papsin, B.C. Sinonasal disease in cystic fibrosis: Clinical characteristics, diagnosis, and management. Pediatr. Pulmonol. 2000, 30, 481-489. [CrossRef]

9. Van Crombruggen, K.; Zhang, N.; Gevaert, P.; Tomassen, P.; Bachert, C. Pathogenesis of chronic rhinosinusitis: Inflammation. J. Allergy Clin. Immunol. 2011, 128, 728-732. [CrossRef]

10. Hulka, G.F. Head and neck manifestations of cystic fibrosis and ciliary dyskinesia. Otolaryngol. Clin. N. Am. 2000, 33, 1333-1341. [CrossRef]

11. Ciofu, O.; Johansen, H.K.; Aanaes, K.; Wassermann, T.; Alhede, M.; von Buchwald, C.; Hoiby, N. P. aeruginosa in the paranasal sinuses and transplanted lungs have similar adaptive mutations as isolates from chronically infected CF lungs. J. Cyst. Fibros. 2013, 12, 729-736. [CrossRef]

12. Hansen, S.K.; Rau, M.H.; Johansen, H.K.; Ciofu, O.; Jelsbak, L.; Yang, L.; Folkesson, A.; Jarmer, H.Ø.; Aanaes, K.; von Buchwald, C.; et al. Evolution and diversification of Pseudomonas aeruginosa in the paranasal sinuses of cystic fibrosis children have implications for chronic lung infection. ISME J. 2011, 6, 31-45. [CrossRef] [PubMed]

13. Mainz, J.G.; Naehrlich, L.; Schien, M.; Kading, M.; Schiller, I.; Mayr, S.; Schneider, G.; Wiedemann, B.; Wiehlmann, L.; Cramer, N.; et al. Concordant genotype of upper and lower airways P. aeruginosa and S. aureus isolates in cystic fibrosis. Thorax 2009, 64, 535-540. [CrossRef]

14. Muhlebach, M.S.; Miller, M.B.; Moore, C.; Wedd, J.P.; Drake, A.F.; Leigh, M.W. Are lower airway or throat cultures predictive of sinus bacteriology in cystic fibrosis? Pediatr. Pulmonol. 2006, 41, 445-451. [CrossRef] [PubMed] 
15. Møller, M.E.; Alanin, M.C.; Grønhøj, C.; Aanœs, K.; Høiby, N.; von Buchwald, C. Sinus bacteriology in patients with cystic fibrosis or primary ciliary dyskinesia: A systematic review. Am. J. Rhinol. Allergy 2017, 31, 293-298. [CrossRef] [PubMed]

16. Roby, B.B.; McNamara, J.; Finkelstein, M.; Sidman, J. Sinus surgery in cystic fibrosis patients: Comparison of sinus and lower airway cultures. Int. J. Pediatr. Otorhinolaryngol. 2008, 72, 1365-1369. [CrossRef]

17. Courtney, J.; Ennis, M.; Elborn, J. Cytokines and inflammatory mediators in cystic fibrosis. J. Cyst. Fibros. 2004, 3, $223-231$. [CrossRef] [PubMed]

18. Babior, B.M. The respiratory burst of phagocytes. J. Clin. Investig. 1984, 73, 599-601. [CrossRef]

19. Brown, R.K.; Kelly, F.J. Role of free radicals in the pathogenesis of cystic fibrosis. Thorax 1994, 49, 738-742. [CrossRef]

20. Day, B.J.; van Heeckeren, A.M.; Min, E.; Velsor, L.W. Role for cystic fibrosis transmembrane conductance regulator protein in a glutathione response to bronchopulmonary Pseudomonas infection. Infect. Immun. 2004, 72, 2045-2051. [CrossRef] [PubMed]

21. Kleme, M.-L.; Levy, E. Cystic fibrosis-related oxidative stress and intestinal lipid disorders. Antioxid. Redox Signal. 2015, 22, 614-631. [CrossRef]

22. Sagel, S.D.; Accurso, F.J. Monitoring inflammation in CF: Cytokines. Clin. Rev. Allergy Immunol. 2002, 23, 041-058. [CrossRef]

23. Downey, D.G.; Bell, S.; Elborn, J. Neutrophils in cystic fibrosis. Thorax 2008, 64, 81-88. [CrossRef]

24. Suter, S.; Schaad, U.B.; Tegner, H.; Ohlsson, K.; Desgrandchamps, D.; Waldvogel, F.A. Levels of free granulocyte elastase in bronchial secretions from patients with cystic fibrosis: Effect of antimicrobial treatment against Pseudomonas aeruginosa. J. Infect. Dis. 1986, 153, 902-909. [CrossRef]

25. Vogelmeier, C.; Hubbard, R.C.; Fells, G.A.; Schnebli, H.P.; Thompson, R.C.; Fritz, H.; Crystal, R.G. Anti-neutrophil elastase defense of the normal human respiratory epithelial surface provided by the secretory leukoprotease inhibitor. J. Clin. Investig. 1991, 87, 482-488. [CrossRef]

26. Beiersdorf, N.; Schien, M.; Hentschel, J.; Pfister, W.; Markert, U.R.; Mainz, J.G. Soluble inflammation markers in nasal lavage from CF patients and healthy controls. J. Cyst. Fibros. 2013, 12, 249-257. [CrossRef]

27. Hentschel, J.; Jäger, M.; Beiersdorf, N.; Fischer, N.; Doht, F.; Michl, R.K.; Lehmann, T.; Markert, U.R.; Böer, K.; Keller, P.; et al. Dynamics of soluble and cellular inflammatory markers in nasal lavage obtained from cystic fibrosis patients during intravenous antibiotic treatment. BMC Pulm. Med. 2014, 14, 82. [CrossRef]

28. Janhsen, W.K.; Arnold, C.; Hentschel, J.; Lehmann, T.; Pfister, W.; Baier, M.; Böer, K.; Hünniger, K.; Kurzai, O.; Hipler, U.-C.; et al . Colonization of CF patients' upper airways with $S$. aureus contributes more decisively to upper airway inflammation than $P$. aeruginosa. Med. Microbiol. Immunol. 2016, 205, 485-500. [CrossRef] [PubMed]

29. Neely, J.G.; Harrison, G.M.; Jerger, J.F.; Greenberg, S.D.; Presberg, H. The otolaryngologic aspects of cystic fibrosis. Trans. Am. Acad. Ophthalmol. Otolaryngol. Am. Acad. Ophthalmol. Otolaryngol. 1972, 76, 313-324.

30. Eggesbø, H.B.; Ringertz, S.; Haanaes, O.C.; Dølvik, S.; Erichsen, A.; Stiris, M.; Kolmannskog, F. CT and MR imaging of the paranasal sinuses in cystic fibrosis. Correlation with microbiological and histopathological results. Acta Radiol. 1999, 40, 154-162. [CrossRef] [PubMed]

31. Hogardt, M.; Kahl, B.C.; Besier, S.; Steinmann, J.; Abele-Horn, M.; Richter, E.; Jäger, G.; Smaczny, C.; Sedlacek, L. Miq 24: Atemwegsinfektionen bei Mukoviszidose. In Mikrobiologisch-Infektiologische Qualitätsstandards, 2nd ed.; Urban \& Fischer: Munich, Germany, 2019.

32. Gehrig, S.; Mall, M.A.; Schultz, P.D.C. Spatially resolved monitoring of neutrophil elastase activity with ratiometric fluorescent reporters. Angew. Chem. Int. Ed. 2012, 51, 6258-6261. [CrossRef]

33. Frey, D.L.; Guerra, M.; Mall, M.A.; Schultz, C. Monitoring neutrophil elastase and cathepsin g activity in human sputum samples. J. Vis. Exp. 2021, 171, e62193. [CrossRef]

34. Quanjer, P.; Stanojevic, S.; Cole, T.; Baur, X.; Hall, G.; Culver, B.; Enright, P.; Hankinson, J.L.; Ip, M.S.; Zheng, J.; et al. Multi-ethnic reference values for spirometry for the 3-95-yr age range: The global lung function 2012 equations. Eur. Respir. J. 2012, 40, 1324-1343. [CrossRef] [PubMed]

35. Singer, F.; Kieninger, E.; Abbas, C.; Yammine, S.; Fuchs, O.; Proietti, E.; Regamey, N.; Casaulta, C.; Frey, U.; Latzin, P. Practicability of nitrogen multiple-breath washout measurements in a pediatric cystic fibrosis outpatient setting. Pediatr. Pulmonol. 2013, 48, 739-746. [CrossRef] [PubMed]

36. Stahl, M.; Joachim, C.; Wielpütz, M.O.; Mall, M.A. Comparison of lung clearance index determined by washout of N2 and SF6 in infants and preschool children with cystic fibrosis. J. Cyst. Fibros. 2019, 18, 399-406. [CrossRef]

37. Anagnostopoulou, P.; Latzin, P.; Jensen, R.; Stahl, M.; Harper, A.; Yammine, S.; Usemann, J.; Foong, R.E.; Spycher, B.; Hall, G.; et al. Normative data for multiple breath washout outcomes in school-aged Caucasian children. Eur. Respir. J. 2019, 55, 1901302. [CrossRef] [PubMed]

38. Hentschel, J.; Müller, U.; Doht, F.; Fischer, N.; Böer, K.; Sonnemann, J.; Hipler, C.; Hünniger, K.; Kurzai, O.; Markert, U.R.; et al. Influences of nasal lavage collection-, processing- and storage methods on inflammatory marker-Evaluation of a method for non-invasive sampling of epithelial lining fluid in cystic fibrosis and other respiratory diseases. J. Immunol. Methods 2014, 404, 41-51. [CrossRef]

39. Fischer, N.; Hentschel, J.; Markert, U.R.; Keller, P.; Pletz, M.; Mainz, J.G. Non-invasive assessment of upper and lower airway infection and inflammation in CF patients. Pediatr. Pulmonol. 2014, 49, 1065-1075. [CrossRef]

40. Jaudszus, A.; Arnold, C.; Hentschel, J.; Hunniger, K.; Baier, M.; Mainz, J.G. Increased cytokines in cystic fibrosis patients' upper airways during a new P. aeruginosa colonization. Pediatr. Pulmonol. 2018, 53, 881-887. [CrossRef] 
41. Elizur, A.; Cannon, C.L.; Ferkol, T.W. Airway inflammation in cystic fibrosis. Chest 2008, 133, 489-495. [CrossRef] [PubMed]

42. Janoff, A.; White, R.; Carp, H.; Harel, S.; Dearing, R.; Lee, D. Lung injury induced by leukocytic proteases. Am. J. Pathol. 1979, 97, 111-136.

43. Johnson, D.; Travis, J. The oxidative inactivation of human alpha-1-proteinase inhibitor. Further evidence for methionine at the reactive center. J. Biol. Chem. 1979, 254, 4022-4026. [CrossRef]

44. Carp, H.; Janoff, A. Potential mediator of inflammation. Phagocyte-derived oxidants suppress the elastase-inhibitory capacity of alpha 1-proteinase inhibitor In Vitro. J. Clin. Investig. 1980, 66, 987-995. [CrossRef]

45. Zhang, Z.; Adappa, N.D.; Lautenbach, E.; Chiu, A.G.; Doghramji, L.J.; Cohen, N.A.; Palmer, J.N. Coagulase-negative staphylococcus culture in chronic rhinosinusitis. Int. Forum. Allergy Rhinol. 2015, 5, 204-213. [CrossRef]

46. Berkhout, M.C.; Klerx-Melis, F.; Fokkens, W.J.; Nuijsink, M.; Van Aalderen, W.M.C.; Heijerman, H.G.M. CT-abnormalities, bacteriology and symptoms of sinonasal disease in children with Cystic Fibrosis. J. Cyst. Fibros. 2016, 15, 816-824. [CrossRef] [PubMed]

47. Eggesbø, H.B.; Eken, T.; Eiklid, K.; Kolmannskog, F. Hypoplasia of the sphenoid sinuses as a diagnostic tool in cystic fibrosis. Acta Radiol. 1999, 40, 479-485. [CrossRef]

48. Eggesbo, H.B.; Sovik, S.; Dolvik, S.; Eiklid, K.; Kolmannskog, F. CT characterization of developmental variations of the paranasal sinuses in cystic fibrosis. Acta Radiol. 2001, 42, 482-493. [CrossRef] [PubMed]

49. Wielpütz, M.O.; von Stackelberg, O.; Stahl, M.; Jobst, B.J.; Eichinger, M.; Puderbach, M.U.; Naehrlich, L.; Barth, S.; Schneider, C.; Kopp, M.V.; et al. Multicentre standardisation of chest MRI as radiation-free outcome measure of lung disease in young children with cystic fibrosis. J. Cyst. Fibros. 2018, 17, 518-527. [CrossRef]

50. Rosenow, T.; Oudraad, M.C.; Murray, C.P.; Turkovic, L.; Kuo, W.; de Bruijne, M.; Ranganathan, S.C.; Tiddens, H.A.; Stick, S.M. Fibrosis australian respiratory early surveillance team for cystic. Pragma-CF. A quantitative structural lung disease computed tomography outcome in young children with cystic fibrosis. Am. J. Respir. Crit. Care Med. 2015, 191, 1158-1165. [CrossRef]

51. Browne, J.P.; Hopkins, C.; Slack, R.; Cano, S.J. The sino-nasal outcome test (SNOT): Can we make it more clinically meaningful? Otolaryngol. Neck Surg. 2007, 136, 736-741. [CrossRef] [PubMed]

52. DeConde, A.S.; Mace, J.C.; Bodner, T.; Hwang, P.H.; Rudmik, L.; Soler, Z.M.; Smith, T.L. SNOT-22 quality of life domains differentially predict treatment modality selection in chronic rhinosinusitis. Int. Forum Allergy Rhinol. 2014, 4, 972-979. [CrossRef] [PubMed] 